\title{
Myxedema Psychosis after Levothyroxine Withdrawal in Radioactive lodine Treatment of Differentiated Thyroid Cancer: A Case Report
}

\author{
Nutnicha Pattaravimonporn ${ }^{a}$ Thanat Chaikijurajai ${ }^{a, b}$ \\ Wichana Chamroonrat ${ }^{c}$ Chutintorn Sriphrapradang ${ }^{a}$ \\ aDepartment of Medicine, Faculty of Medicine Ramathibodi Hospital, Mahidol University, \\ Bangkok, Thailand; 'b Internal Medicine Residency Program, University of Minnesota Medical \\ School, Minneapolis, MN, USA; 'Division of Nuclear Medicine, Department of Radiology, \\ Faculty of Medicine Ramathibodi Hospital, Mahidol University, Bangkok, Thailand
}

\section{Keywords}

Hypothyroidism · Psychotic disorders · Radioiodine $\cdot$ Thyroidectomy $\cdot$ Thyroxine

\begin{abstract}
Neuropsychiatric symptoms, especially acute psychosis (often referred to as myxedema madness or psychosis), are rare but possible clinical presentations of patients with hypothyroidism. A 42-year-old woman with papillary thyroid carcinoma and recent total thyroidectomy had developed flat affect, paranoid delusion, and visual and auditory hallucination during inpatient admission for elective radioactive iodine treatment. On admission, her history and physical exam did not reveal symptoms and signs of significant hypothyroidism. Other medical causes of acute psychosis were excluded, and the patient was immediately treated with thyroid hormone replacement therapy. Subsequently, her thyroid function normalized, and her psychotic symptoms gradually improved. Although there is a lack of classic signs and symptoms of hypothyroidism, myxedema madness should be recognized as one of the potentially treatable causes of acute psychosis.
\end{abstract}


Pattaravimonporn et al.: Myxedema Psychosis

\section{Introduction}

Hypothyroidism is a common condition affecting up to $10 \%$ of the adult general population [1] and can have an impact on almost every organ system in the body. Common classic clinical presentation of hypothyroidism includes fatigue, unintentional weight gain, dry skin, edema, myalgia, constipation, cold intolerance, and hoarseness [1]. Considering that most of the symptoms and signs of hypothyroidism are nonspecific, they may vary in presentation and severity among patients. Hypothyroidism is often underdiagnosed, especially in elderly female patients [2]. Moreover, other less common neuropsychiatric symptoms, such as psychomotor retardation, depression, cognitive impairment, and dementia, can also be found in patients with hypothyroidism [1]. Although the role of thyroid hormone in the regulation of mood, behavior, and cognitive function has been widely recognized, the potential impact of thyroid hormone imbalance, especially hypothyroidism, on neuropsychiatric function still remains unclear and has not been well studied. One of the rare neuropsychiatric manifestations of hypothyroidism is psychosis, often referred to as myxedema madness or myxedema psychosis. In the present study, we hereby present a case of a patient who developed myxedema madness without presenting any classic signs or symptoms of hypothyroidism.

\section{Case Presentation}

A 42-year-old woman with papillary thyroid carcinoma and intermediate risk of recurrence underwent total thyroidectomy 7 weeks prior to an elective radioactive iodine (RAI) treatment; therefore, thyroid hormone replacement therapy was withheld. Her preadmission thyroid-stimulating hormone (TSH) level was over $100 \mathrm{mIU} / \mathrm{mL}$. On the first day of admission, she was found to have flat affect, paranoid delusion, and visual and auditory hallucinations. She believed that her sister and hospital staffs were trying to harm her. She refused the RAI treatment and tried to leave the hospital. In retrospect, she reported her concern about her health and did not sleep well for 2 weeks prior to admission. She believed that there were devils in her house and her sister cast a spell over her. She also stated that she could foresee things in the future and hear threatening voices. She had no history of mental illness. Physical examination and baseline laboratory findings (i.e., complete blood count, electrolytes, liver function test, creatinine, and plasma glucose levels) were unremarkable, except for the TSH level of over $100 \mathrm{mIU} / \mathrm{mL}$ and a high thyroglobulin level of $73.4 \mathrm{ng} / \mathrm{mL}$. Her mental status examination was normal, except for concentration deficit. Therefore, a psychiatry consultation was made, and the RAI treatment was deferred. She was diagnosed with hypothyroidisminduced psychosis and was commenced on oral haloperidol, quetiapine, and levothyroxine $100 \mu \mathrm{g}$ daily. With multidisciplinary agreement, she was discharged from the RAI treatment unit and managed her psychotic symptoms and hypothyroidism in an outpatient setting. At her follow-up visit 2 weeks later, she reported that she no longer had any visual or auditory hallucination and became less paranoid. Her TSH level was still over $100 \mathrm{mIU} / \mathrm{mL}$; however, her free T4 level was within normal ranges of $0.84 \mathrm{ng} / \mathrm{dL}$ (reference range, $0.7-1.48 \mathrm{ng} / \mathrm{dL}$ ). At 2 months after discharge, her TSH level was decreased to $0.1 \mathrm{mIU} / \mathrm{mL}$, along with increased free T3 and free T4 levels with the levels of $4.12 \mathrm{ng} / \mathrm{dL}$ (reference range, $1.88-3.18 \mathrm{pg} / \mathrm{mL}$ ) and $1.81 \mathrm{ng} / \mathrm{dL}$, respectively. Upon several follow-up visits, her symptoms continued to improve and finally resolved with her TSH and free thyroid hormone levels being consistently within the appropriate ranges. She was then re-scheduled for the RAI treatment with the use of recombinant human TSH (rhTSH) instead of levothyroxine withdrawal protocol. She had an uneventful RAI treatment with rhTSH protocol 4 months after the first attempt.

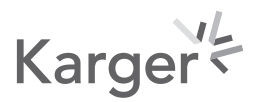


Pattaravimonporn et al.: Myxedema Psychosis

\section{Discussion}

The term "myxedematous madness" was first described in 1949 by Richard Asher [3], who reviewed 14 cases of hypothyroidism associated with acute psychosis. Subsequently, even though a number of cases with myxedema psychosis have been continuously reported [4,5], hypothyroidism is still one of the most common missed causes of organic psychosis [6,7]. It might be caused by a lack of specific characteristics of psychotic symptoms, diagnostic criteria, correlation with the severity of hypothyroidism, and different responses to thyroid hormone supplement, thereby making the thyroid function test a routine laboratory investigation in patients with psychosis [7].

In patients with new-onset psychosis, the most important initial diagnostic evaluation is to rule out possible organic or secondary causes of psychosis. Some factors and features of psychotic symptoms are suggestive of medical causes rather than primary psychiatric disorders [8]. For instance, acute onset is known to be suggestive of a substance-induced or medical cause of psychosis, which was found in this patient given that her symptoms started 2 weeks before the admission. Older age at onset, usually 40 years or older, is more common in patients with organic psychosis. Nonauditory hallucination is also suggestive of secondary causes of psychosis. However, this patient presented with both visual and auditory hallucination, which did not help much with differentiating secondary from primary psychosis. In addition, a lack of prodromal symptoms and family history of psychiatric disorders in this patient was also another clue suggesting medical psychiatric causes.

In general, the diagnosis of myxedema psychosis relies on the presence of hypothyroidism in the thyroid function test, as well as the exclusion of other secondary causes of psychosis. It is often seen in patients with overt hypothyroidism from Hashimoto thyroiditis [9] or thyroid cancer after a thyroidectomy [10], which was the case in this patient. Interestingly, the classic signs and symptoms of hypothyroidism may not present in patients with myxedema madness [5]. In terms of imaging investigations, white matter changes in the frontal lobe on a brain magnetic resonance imaging [4] and a generalized decrease in cerebral blood flow on singlephoton emission computed tomography studies $[11,12]$ have also been reported. Gupta et al. [13] also revealed a reversible decrease in overall wave activity on the electroencephalogram in a patient with suspected myxedema psychosis. Nevertheless, the sensitivity and specificity of these imaging techniques in diagnosing myxedema psychosis have never been studied. Response to thyroid hormone supplement could also be helpful for confirming the diagnosis [14], but some reports suggested that psychiatric symptoms may not respond well to thyroid hormone replacement $[6,9]$. In this patient, a history of thyroidectomy, her age, the onset of the psychotic symptoms, a lack of prodromal period, and other possible secondary causes of acute psychosis, as well as an impressive response to thyroid hormone replacement therapy, all made myxedema psychosis the most likely diagnosis. Interestingly, given that our patient subsequently underwent an eventful RAI treatment with the use of rhTSH, this strategy seems to help prevent myxedema psychosis, which supports the previous report on TSH replacement being used as an alternative to levothyroxine withdrawal in patients with thyroid cancer who have a history of myxedema psychosis and require RAI ablation or treatment [10].

\section{Conclusions}

When evaluating patients with psychosis, hypothyroidism should be ruled out as one of the possible secondary causes of psychosis, especially in patients with a history of thyroid problems or thyroidectomy regardless of the presence of other signs and symptoms of hypothyroidism or the level of T3 and T4 in the thyroid function test. There is no specific pattern

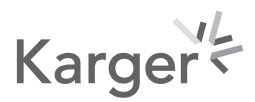


of psychosis or specific imaging or laboratory investigation. Furthermore, following thyroid hormone replacement therapy, psychiatric symptoms may persist or take longer than systemic symptoms to respond. One of the possible approaches to preventing myxedema psychosis in patients with thyroid cancer is the use of rhTSH for increasing TSH levels before undergoing RAI ablation or treatment.

\section{Statement of Ethics}

Written informed consent was obtained from the patient for publication of this case report. This case report was reviewed and approved by the Human Research Ethics Committee, Faculty of Medicine Ramathibodi Hospital, Mahidol University (MURA2021/691). The authors confirmed that they strictly followed the guideline of 1964 Declaration of Helsinki and related good clinical practices in writing this case report. The patient's identifiable information and identity were completely anonymized.

\section{Conflict of Interest Statement}

The authors have no conflicts of interest to declare.

\section{Funding Sources}

The authors did not receive any financial support.

\section{Author Contributions}

N.P. and T.C. were involved in writing the draft, which was further modified after critical inputs from W.C. and C.S. All the authors agreed upon the final version of the manuscript.

\section{Data Availability Statement}

All data generated or analyzed during this study are included in this article. Further enquiries can be directed to the corresponding author.

\section{References}

1 Chaker L, Bianco AC, Jonklaas J, Peeters RP. Hypothyroidism. Lancet. 2017;390(10101):1550-62.

2 Carlé A, Pedersen IB, Knudsen N, Perrild H, Ovesen L, Andersen S, et al. Hypothyroid symptoms fail to predict thyroid insufficiency in old people: a Population-Based Case-Control Study. Am J Med. 2016;129(10):1082-92.

3 Asher R. Myxoedematous madness. Br Med J. 1949;2(4627):555-62.

4 Kruger J, Kraschewski A, Jockers-Scherubl MC. Myxedema madness: systematic literature review of published case reports. Gen Hosp Psychiatry. 2021 Aug 14;72:102-16.

5 Mohamed MFH, Danjuma M, Mohammed M, Mohamed S, Siepmann M, Barlinn K, et al. Myxedema psychosis: systematic review and pooled analysis. Neuropsychiatr Dis Treat. 2021;17:2713-28.

6 Azzopardi L, Murfin C, Sharda A, De Silva N. Myxoedema madness. BMJ Case Rep. 2010;2010:bcr0320102841.

7 Endres D, Matysik M, Feige B, Venhoff N, Schweizer T, Michel M, et al. Diagnosing organic causes of schizophrenia spectrum disorders: findings from a one-year cohort of the Freiburg Diagnostic Protocol in Psychosis (FDPP). Diagnostics. 2020;10(9):691. 
8 Freudenreich 0, Schulz SC, Goff DC. Initial medical work-up of first-episode psychosis: a conceptual review. Early Interv Psychiatry. 2009;3(1):10-8.

9 Heinrich TW, Grahm G. Hypothyroidism presenting as psychosis: myxedema madness revisited. Prim Care Companion J Clin Psychiatry. 2003;5(6):260-6.

10 Morosán Allo YJ, Rosmarin M, Urrutia A, Faingold MC, Musso C, Brenta G. Myxedema madness complicating postoperative follow-up of thyroid cancer. Arch Endocrinol Metab. 2015 Aug;59(4):359-63.

11 Kinuya S, Michigishi T, Tonami N, Aburano T, Tsuji S, Hashimoto T. Reversible cerebral hypoperfusion observed with Tc-99m HMPAO SPECT in reversible dementia caused by hypothyroidism. Clin Nucl Med. 1999; 24(9):666-8.

12 Constant EL, de Volder AG, Ivanoiu A, Bol A, Labar D, Seghers A, et al. Cerebral blood flow and glucose metabolism in hypothyroidism: a Positron Emission Tomography Study. J Clin Endocrinol Metab. 2001;86(8):3864-70.

13 Gupta SP, Gupta PC, Kumar V, Ahuja MM. Electroencephalographic changes in hypothyroidism. Indian J Med Res. 1972 Jul;60(7):1101-6.

14 Cook DM, Boyle PJ. Rapid reversal of myxedema madness with triiodothyronine. Ann Intern Med. 1986 Jun; 104(6):893-4. 